\title{
Predicting the efficiency of deposit removal during filter backwash
}

\author{
BM Brouckaert ${ }^{1 *}$, A Amirtharajah ${ }^{2}$, CJ Brouckaert ${ }^{1}$ and JE Amburgey ${ }^{3}$ \\ ${ }^{1}$ Pollution Research Group, School of Chemical Engineering, University of KwaZulu-Natal, Durban 4041, South Africa \\ ${ }^{2} \mathrm{CH} 2 \mathrm{M}$-Hill, Sacramento, CA, USA \\ ${ }^{3}$ Department of Civil Engineering, University of North Carolina at Charlotte, Charlotte, NC, USA
}

\begin{abstract}
The long-term performance of granular media filters used in drinking water treatment is ultimately limited by the efficiency of the backwash process. This paper demonstrates that it is possible to develop quantitative predictions of backwash efficiency based on filter operating conditions. An experimental investigation into the effect of backwash rate, type of coagulant, degree of clogging and accumulation of residual deposits (not removed by backwash) on the efficiency of fluidised bed filter backwash in laboratory scale filters is described. A natural raw water was used and small variations in the raw water characteristics (manifested as variations in raw water turbidity, temperature, $\mathrm{pH}$, rate of head loss development and turbidity removal efficiency) within each set of experiments appeared to affect the efficiency of backwash in addition to the parameters varied deliberately. Stepwise linear regression and statistical analysis of model significance were used to determine which of several possible filtration and backwash parameters were the best predictors of backwash performance. Backwash rate, filter run time, rate of head loss development and mass of residual deposits accumulated during previous runs were found to be the best predictors of backwash efficiency for any given filter cycle. Floc deposits appeared to become more difficult to remove the longer they remained in the filter, while rate of head loss development appeared to provide some indication of the strength of cohesive deposits for filter runs of similar length. The efficiency of detachment of freshly deposited floc appeared to increase as the mass of residual deposits and mud balls in the filter increased. The numerical correlations developed in this study are site specific but the methodology can be adapted to any filter operation and backwash regime.
\end{abstract}

Keywords: water treatment, sand filters, fluidised backwash, backwash efficiency, backwash modelling, floc ageing

\section{Introduction}

Failure to adequately clean filters during backwashing results in the deterioration of the state of the filter bed, which can eventually impair the performance of the filter. Filters with inefficient backwash tend to accumulate aggregates of dirt, media and coagulant known as mudballs (Logsdon et al., 2002). These can grow into inactive sub-surface masses of clogged material which increase local velocities in the filter with a potentially negative impact on filtrate turbidity and filter run time (Cleasby, 1990). Larger mud balls can sink into the fluidised media and accumulate adjacent to the underdrain and gravel layers, causing nonuniform distribution of the backwash water. Clogged regions of the filter also tend to contract as the head loss increases, leading to the development of cracks in the bed, which result in short circuiting of the filter influent and a subsequent decline in filtered water quality (Kawamura, 1975; Cleasby, 1990).

Over the past few decades, there has been a substantial amount of research on modelling the filtration phase of the filter operating cycle. However, there has been little fundamental research into the backwash process and few of the models developed can easily be adapted for use in full scale water treatment plants. A critical review of previous approaches to backwash modelling is presented in Brouckaert (2004). While a number of

This paper was originally presented at the 2006 Water Institute of South Africa (WISA) Biennial Conference, Durban, South Africa, 21-25 May 2006.

* To whom all correspondence should be addressed.

皿+2731 260-3375; fax:+2731 260-3241; e-mail: brouckaertb@ukzn.ac.z backwash studies have yielded important insights into the mechanisms involved in filter cleaning (Amirtharajah, 1978; Amirtharajah and Giourgas, 1981; Fitzpatrick, 1993; Mahmood et al., 1998) and have made it possible to predict the conditions for optimum backwash (Amirtharajah, 1978; Amirtharajah, 1984), none has led to the development of a model which can predict what the actual backwash efficiency will be under a given set of conditions and how this will affect the filter in the long term.

In order for any model of filter backwash efficiency to be useful for the design and optimisation of filters outside of a closely controlled laboratory environment, it is important that it includes all relevant processes and effects, and that the input data required are readily available at most treatment plants. A better understanding of backwashing would assist in the optimisation of existing filters as well as improving filter design for more robust operation, particularly in applications where optimum backwash cannot be guaranteed. These applications include many water treatment plants in developing countries which operate without auxiliary wash (air scour or surface wash).

This paper presents experimental results and analysis of the impact of various factors on backwash effectiveness and their implications for the operation of real filters. The experimental work was restricted to fluidised bed backwash but the approach taken in modelling and analysing the data should be equally applicable to auxiliary backwash systems.

\section{Theoretical considerations}

At the scale of a single floc coated filter grain, the probability that some or all of the floc deposit will detach during back- 
wash depends on the relative magnitudes of the detachment and attachment forces as well as the duration of the backwash. This can be expressed mathematically as:

$$
-\int_{0}^{t} \frac{\partial \sigma}{\partial t} d t=\int_{0}^{t} f_{1}(\sigma) \cdot f_{2}\left(\frac{F_{D}}{F_{A}}\right) d t
$$

where:

$\sigma=$ Specific deposit, $\mathrm{g}$ deposit $/ \mathrm{cm}^{3}$ of filter grains

$t=$ Backwash time, $\mathrm{s}$

$F_{D} \quad=$ Characteristic detachment force

$F_{A}=$ Characteristic attachment force

$f_{l}^{A}(\sigma)=$ Function of the amount and properties of the floc still attached to filter grains, $g$ deposit $/ \mathrm{cm}^{3}$

$f_{2}\left(\frac{F_{D}}{F_{A}}\right)=\begin{aligned} & \text { of filter grains } \\ & \text { Probability that at any given floc aggregate will }\end{aligned}$ be detached at time $\mathrm{t}, \mathrm{s}^{-1}$

In practice, filter backwash is usually continued until the backwash effluent is relatively clean, i.e. until the amount of detachment still occurring becomes essentially negligible. The total mass of deposits present in the filter at the end of the filter run (before backwash) can therefore be divided into two fractions: a fraction which is detachable under given backwash conditions and a fraction which is not. Given an adequate duration of backwash, it is reasonable to assume that the mass of deposits which will not detach is primarily a function of hydrodynamic conditions (represented in Eq. [1] by the characteristic detachment force, $F_{D}$ ) and the properties of the floc deposits (represented by $F_{A}$ ).

One possible source of information about the strength and adhesiveness of floc deposits (i.e. factors contributing to $F_{A}$ ) is operating and performance data collected during the filtration phase of the filter cycle. The more easily floc becomes attached to the media during filtration (i.e. the stronger the attachment forces), the more difficult it may be to detach during backwash. Indeed, it is generally known that factors such as smaller media sizes and the use of polymers in the filter influent which tend to improve filtrate quality also tend to promote mud-balling (Kawamura, 1975; Cleasby, 1990). Consequently, it may be possible to correlate backwash efficiency with certain aspects of filter design and performance. Establishing which filtration and backwash parameters are the best predictors of backwash performance could also help to elucidate some of the fundamental mechanisms involved.

\section{Experimental work}

This paper presents the results of three sets of filter backwash experiments that investigated the effect of various parameters on backwash performance. Backwash performance was evaluated by determining the mass of residual deposits, $M_{R}$, retained in the filter after backwash (in this case, fluidised bed backwash) was complete. $M_{R}$ was expressed in units of $\mathrm{g} / \mathrm{m}^{2}$ cross-sectional filter area. The filter area is the area perpendicular to the flow. The first two sets of experiments, referred to as the $A U$ and $Z U$ experiments, were designed to investigate the effect of type of coagulant used, degree of filter loading and filter backwash rate on backwash performance. The last set of experiments, referred to as the $M$ experiments, was designed to determine the rate of accumulation of retained mass over consecutive filter runs with fluidised backwash. The following sections describe the experimental equipment and procedures used.

\section{Laboratory filters}

The experiments were carried out in two $20 \mathrm{~cm}$ diameter laboratory filters at the Umgeni Water Process Evaluation Facility in Durban, South Africa. A detailed description of the filters is provided in Brouckaert (2004). Raw water was drawn from the head of the Wiggins Water Treatment Plant which treats water from the Inanda Dam.

Experiments to measure the accumulation of mud over multiple filter cycles were carried out in a filter with $0.64 \mathrm{~m}$ of 0.7 $\mathrm{mm}$ sand (effective size $0.72 \mathrm{~mm}$, uniformity coefficient 1.36). Experiments on the effect of backwash rate and degree of clogging on backwash performance were carried out using a filter bed consisting of $0.47 \mathrm{~m}$ of sand sieved between 1 and $1.4 \mathrm{~mm}$ (effective size $1.04 \mathrm{~mm}$, uniformity coefficient 1.22).

\section{Experimental procedures}

Filtration rate, temperature and head loss profile were recorded during the course of each run. Grab samples of the raw water, coagulated influent water and filtrate were collected at intervals and analysed for turbidity and $\mathrm{pH}$. The total volume of water filtered during each run was calculated from the flow rate measurements.

At the end of each filter run, the filter was backwashed with a pre-determined backwash rate and volume $\left(4.27 \mathrm{~m}^{3} / \mathrm{m}^{2}\right.$ filter area for the 1 to $1.4 \mathrm{~mm}$ sand bed and $3.18 \mathrm{~m}^{3} / \mathrm{m}^{2}$ for the $0.7 \mathrm{~mm}$ sand bed) using tap water. The backwash effluent was collected in $120 \ell$ plastic drums. Once backwash was complete, the volume of effluent was measured and then the contents of each drum were stirred to obtain a uniform concentration. Samples of the mixed effluent were collected for turbidity and suspended solids measurements (Standard Methods, 1990). The total mass of solids in the backwash effluent determined from the suspended solids concentration was termed mass detached, $M_{D}$, and expressed in units of $\mathrm{g}$ of suspended solids per $\mathrm{m}^{2}$ cross-sectional filter area.

Floc retained in the bed after fluidised bed backwash was dislodged by multiple cycles of combined air and sub-fluidisation water wash followed by a high rate rinse step. During the combined air and water step, rapid variations in air flow were used to maximise agitation of the bed. Pulsing the air flow resulted in much more violent backwash conditions than can be achieved during collapse pulsing (Amirtharajah, 1984), which is generally held to be the most effective backwash regime available (Cleasby, 1990). Consequently, it was assumed to be more efficient in detaching floc than normal collapse pulsing.

The water level in the filter was drawn down to just above the bed surface before the start of air scour and the air and water flows were shut off before the water reached the backwash trough in order to prevent media losses. The bed was then flushed with water at high rate to remove the detached floc. This process was repeated until the amount of additional floc being removed from the bed became negligible. The effluent from combined air and water wash was collected and analysed in the same way as the fluidised backwash effluent. The mass of floc recovered in this stage was the residual mass or mass retained, $M_{R}\left(\mathrm{~g} / \mathrm{m}^{2}\right)$.

\section{Experiments on the effect of coagulant used, degree of clogging and backwash rate on backwash efficiency for single filter runs}

Two sets of experiments were carried out to investigate the effect of coagulant type, degree of filter clogging and back- 


\begin{tabular}{|c|c|c|c|c|}
\hline \multicolumn{5}{|c|}{$\begin{array}{l}\text { TABLE } 1 \\
\text { Experimental conditions for the } A U \text { and } Z U \text { experiments }\end{array}$} \\
\hline \multirow[b]{2}{*}{$\mathrm{AU}$} & \multirow{2}{*}{$\begin{array}{l}\text { Coagulant } \\
\text { Alum }\end{array}$} & \multirow[b]{2}{*}{$\begin{array}{l}\text { Parameters varied } \\
\text { Backwash rate: } \\
61-70 \mathrm{~m} / \mathrm{h} \\
\\
\text { Terminal head loss: } \\
0.3-1.4 \mathrm{~m}\end{array}$} & \multicolumn{2}{|c|}{ Filter operating conditions } \\
\hline & & & $\begin{array}{l}\text { Dose * } \\
\text { Filtration rate* } \\
\text { Influent turbidity* } \\
\text { Filtrate turbidity* } \\
\text { Temperature* } \\
\text { pH after coagulation* } \\
\text { Filter run time }\end{array}$ & $\begin{array}{l}8-8.3 \mathrm{mg} / \ell \\
6.2-6.7 \mathrm{~m} / \mathrm{h} \\
2.1-2.8 \mathrm{NTU} \\
0.22-0.29 \mathrm{NTU} \\
24.6-25.3^{\circ} \mathrm{C} \\
7.2-7.9 \\
8-32 \mathrm{~h}\end{array}$ \\
\hline ZU & Z464N & $\begin{array}{l}\text { Backwash rate: } \\
61-95 \mathrm{~m} / \mathrm{h}\end{array}$ & $\begin{array}{l}\text { Dose* } \\
\text { Filtration rate* } \\
\text { Influent turbidity* } \\
\text { Filtrate turbidity* } \\
\text { Temperature* } \\
\text { pH after coagulation* } \\
\text { Filter run time }\end{array}$ & $\begin{array}{l}2.5-2.8 \mathrm{mg} / \ell \\
6.2-6.6 \mathrm{~m} / \mathrm{h} \\
0.9-1.3 \mathrm{NTU} \\
0.10-0.13 \mathrm{NTU} \\
21.7-24.6^{\circ} \mathrm{C} \\
7.7-8.4 \\
40-50 \mathrm{~h}\end{array}$ \\
\hline
\end{tabular}

*Average conditions for each run

\begin{tabular}{|c|c|c|c|}
\hline \multicolumn{4}{|c|}{$\begin{array}{c}\text { TABLE } 2 \\
\text { Mass accumulation experiments }\end{array}$} \\
\hline Series & Number of & \multicolumn{2}{|c|}{ Experimental conditions } \\
\hline M0 & 1 & \multirow{7}{*}{\begin{tabular}{|l} 
Coagulant \\
Dose * \\
Filtration rate* \\
Influent turbidity* \\
Filtrate turbidity* \\
Temperature* \\
pH after coagulation* \\
Filter run time (M0, M0D) \\
Filter run time (MxRx) \\
Backwash rate \\
\end{tabular}} & \multirow{7}{*}{$\begin{array}{l}\text { Alum } \\
5.8-6.9 \mathrm{mg} / \ell \\
6.0 \text { to } 6.6 \mathrm{~m} / \mathrm{h} \\
0.8-1.5 \mathrm{NTU} \\
0.15-0.37 \mathrm{NTU} \\
20.5-25.8^{\circ} \mathrm{C} \\
7.4-7.7 \\
26.3-28.5 \mathrm{~h} \\
18.7-24.0 \mathrm{~h} \\
54 \mathrm{~m} / \mathrm{h} \\
\end{array}$} \\
\hline M0D & 1 & & \\
\hline M9R1 - M9R9 & 9 & & \\
\hline M4R1 - M4R4 & 4 & & \\
\hline M1R1 & 1 & & \\
\hline M2R1 - M2R2 & 2 & & \\
\hline M6R1 - M6R6 & 6 & & \\
\hline
\end{tabular}

*Average conditions for each run

wash rate on backwash efficiency for single filter runs. The first set used alum as the coagulant and both the terminal head loss and the backwash rate were varied to determine their effect on backwash efficiency. These experiments are referred to as the $A U$ experiments. The second set of experiments, the $Z U$ experiments, used the coagulant $Z 464 \mathrm{~N}$ (Zetachem, South Africa), a proprietary blend of a polyDADMAC (poly diallyl dimethyl ammonium chloride) and aluminium chlorohydrate used at the Wiggins Water Treatment Works, Durban, South Africa. Table 1 summarises the experimental conditions for the $A U$ and $Z U$ experiments.

The $A U$ series of experiments included 4 runs with the terminal head loss held constant at $1.4 \mathrm{~m}$ while the backwash rate was varied between 61 to $70 \mathrm{~m} / \mathrm{h}$, and an additional three runs where the backwash rate was held constant at $61 \mathrm{~m} / \mathrm{h}$ and the terminal head loss was varied between 0.3 and $1.0 \mathrm{~m}$. A total of $10 \mathrm{ZU}$ experiments were carried out where the terminal head loss was kept in the range 1.6 to $1.75 \mathrm{~m}$ and the backwash rate was varied from 61 to $95 \mathrm{~m} / \mathrm{h}$.

\section{Mass accumulation experiments}

The mass accumulation $(M)$ experiments consisted of four series of consecutive filter runs with fluidised water-only backwash between each run plus three single run experiments. The backwash rate and volume was the same for all experiments but the number of runs per series was varied. The cumulative mass retained $\left(\Sigma M_{R}\right)$ in the filter was measured after the last filter cycle (filtration + fluidised backwash) in each series as described in the previous section. Table 2 shows the number of runs in each series, listed in chronological order. The experimental conditions are also shown. Note that M9R1 refers to the first run in the series of 9 consecutive filter runs.

The target run time for all experiments except M0 and M0D was $\sim 24 \mathrm{~h}$; however, in some cases it was necessary to terminate the run earlier because the maximum available head loss had been reached. The run times for M0 and M0D were 26.3 and 28.5 h respectively.

\section{Data analysis}

During this study, experimental conditions were kept as constant as possible. However, since a natural raw water source was used, some variation in raw water quality was unavoidable and this appeared to affect both filter performance and the effectiveness of backwash. Model selection techniques using multivariate linear regression provide a relatively simple way of determining which of a number of possible parameters are significantly correlated with filter backwash performance and whether the correlations are positive or negative. Multiple linear regression was used to analyse the data from the $A U$ and $Z U$ experiments in order to identify statistically significant 


\begin{tabular}{|c|c|c|}
\hline \multicolumn{3}{|c|}{$\begin{array}{c}\text { TABLE } 3 \\
\text { Parameters included in the regression analysis for } A U \text { and } Z U \text { experiments }\end{array}$} \\
\hline Variable & Description & Units \\
\hline $\begin{array}{l}v_{b} \\
M_{T F} \\
V_{f} \\
h_{f} \\
t_{f} \\
\text { coag } \\
\text { filt } \\
\text { rawpH } \\
\text { filtpH } \\
T \\
v_{f} \\
\text { dose } \\
\text { filt/coag } \\
\left(\text { dh } / d V_{f}\right)\end{array}$ & $\begin{array}{l}\text { Backwash rate } \\
\text { Total mass deposited during filtration (mass detached }+ \text { mass retained) } \\
\text { Total volume filtered } \\
\text { Terminal head loss } \\
\text { Filter run time } \\
\text { Average influent turbidity (coagulated) } \\
\text { Average filtrate turbidity } \\
\text { Raw pH } \\
\text { Filtered water pH } \\
\text { Temperature } \\
\text { Average filtration rate } \\
\text { Average coagulant dose } \\
\text { Ratio of average filtrate to average influent turbidity } \\
\text { Rate of head loss development } \\
\text { ([terminal - initial head loss]/volume filtered) }\end{array}$ & $\begin{array}{l}\mathrm{m} / \mathrm{h} \\
\mathrm{g} / \mathrm{m}^{2} \text { filter area } \\
\mathrm{m}^{3} / \mathrm{m}^{2} \text { filter area } \\
\mathrm{m} \\
\mathrm{h} \\
\text { NTU } \\
\text { NTU }\end{array}$ \\
\hline
\end{tabular}

correlations between backwash performance and experimental conditions of the form:

$$
M_{R}=a_{0}+a_{b 1} p_{b 1}+a_{b 2} p_{b 2}+\ldots+a_{f 1} p_{f 1}+a_{f 2} p_{f 2}+\ldots
$$

where:

$$
\begin{array}{ll}
M_{R} & \begin{array}{l}
\text { Mass retained after fluidised back- } \\
\text { wash } \mathrm{g} / \mathrm{m}^{2} \text { filter area }
\end{array} \\
a_{0}, a_{b 1}, \ldots, a_{f 1}, \ldots= & \text { Regression coefficients } \\
p_{b 1}, p_{b 2}, \ldots . & \begin{array}{l}
\text { Parameters relating to the backwash } \\
\text { phase which reflect the intensity of } \\
\\
\text { backwash forces }
\end{array} \\
p_{f 1}, p_{f 2}, \ldots . \quad \begin{array}{l}
\text { Parameters relating to the filtration } \\
\text { phase which reflect the floc charac- } \\
\text { teristics and degree of clogging of } \\
\text { the filter }
\end{array}
\end{array}
$$

For each set of experiments, first the relationship between mass retained and the control variable(s) (backwash rate for $Z U$ experiments; backwash rate and degree of clogging for $A U$ experiments) was established and then the data was further analysed to identify additional terms in Eq. [2]. Table 3 provides a list of parameters considered in the regression analysis for each set of experiments.

Analysing the results of the mass accumulation experiments was more complicated because the mass retained after each individual cycle (filter run + backwash) in each series of runs was unknown. Overall there appeared to be a fairly steady accumulation of mass retained in the filter with increasing number of runs, however the mass increase per cycle appeared to decline as the number of cycles increased. Therefore, the following model of residual mass accumulation was proposed:

$$
\left(\sum M_{R}\right)_{i}=\left(\sum M_{R}\right)_{i-1}+a_{0}+a_{f 1} p_{f 1}+a_{f 2} p_{f 2}+\ldots-\beta\left(\sum M_{R}\right)_{i-1}
$$

where:

$$
\begin{array}{ll}
\left(\Sigma M_{R}\right)_{i}= & \begin{array}{l}
\text { Cumulative mass retained after the } i^{\text {th }} \\
\text { filter cycle, } \mathrm{g} / \mathrm{m}^{2}
\end{array} \\
a_{0}, a_{f 1}, a_{f 2}, \ldots, \beta= & \text { Fitting parameters }
\end{array}
$$

Statistical analysis was then used to determine which of the parameters listed in Table 3 resulted in a significant improvement in model predictions when included in Eq. [3] (as $p_{f 1}, p_{f 2}$, etc.).

\section{Results and discussion}

\section{Effect of degree of clogging, coagulant used and backwash rate on backwash efficiency for a single filter cycle}

Figures 1 and 2 show the mass retained in the filter for the $A U$ and $Z U$ experiments respectively. The trend line in Fig. 1 was calculated for the 4 experiments with terminal head loss $=1.4 \mathrm{~m}$ and variable backwash rate (circles). The run times for the 4 experiments with backwash rate $=61 \mathrm{~m} / \mathrm{h}$ (triangles) are given in brackets. The two series have one experiment in common.

For both the $Z U$ experiments and the $A U$ experiments with constant terminal head loss, there is a clear correlation between backwash rate and mass retained. In the case of the $Z U$ experiments, the mass retained appeared to approach either a minimum or a plateau at around $95 \mathrm{~m} / \mathrm{h}$. Consequently a quadratic trend line fit the data better than a linear trend line. As expected, coagulation with $\mathrm{Z} 464 \mathrm{~N}$ rather than alum appeared to make backwash less effective at similar backwash rates.

In the $A U$ experiments, the effect of the degree of clogging on the mass retained after backwashing was similar in magnitude to the effect of varying the backwash rate from 61 to $70 \mathrm{~m} / \mathrm{h}$. This suggests that one of the filtration parameters, $p_{f}$, in Eq. [2] should reflect the degree of clogging of the

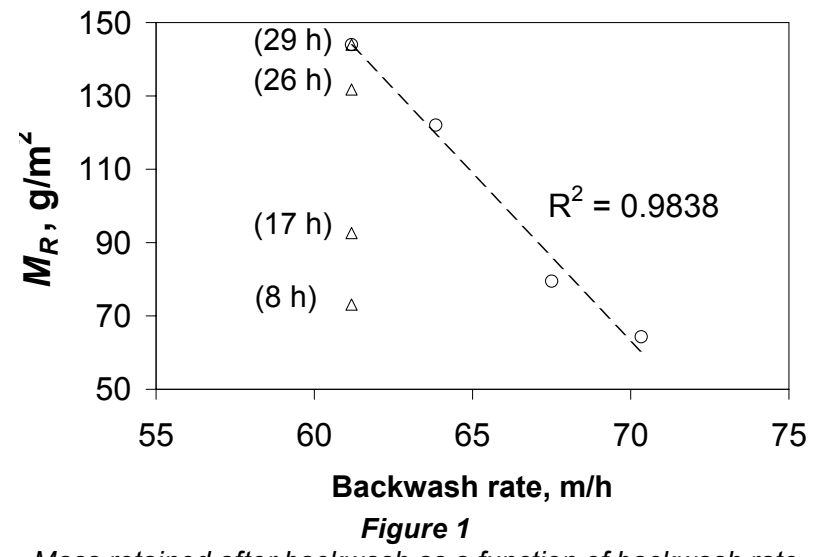

Mass retained after backwash as a function of backwash rate - $A U$ experiments 


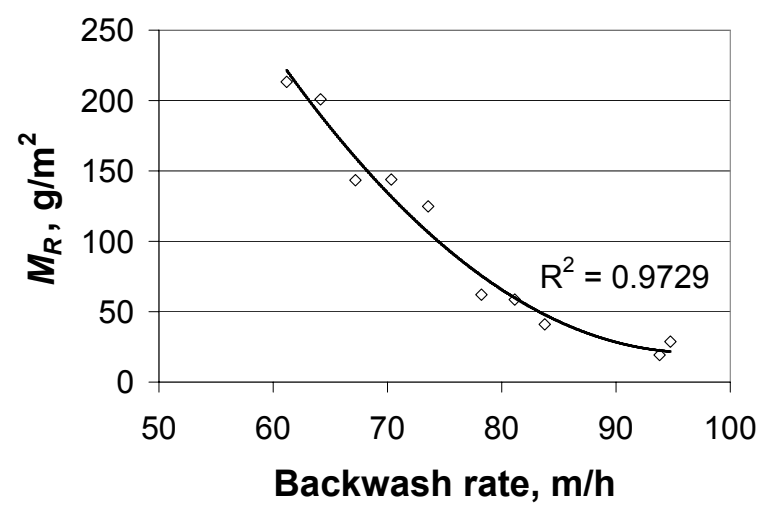

Figure 2

Mass retained after backwash as a function of backwash rate - ZU experiments

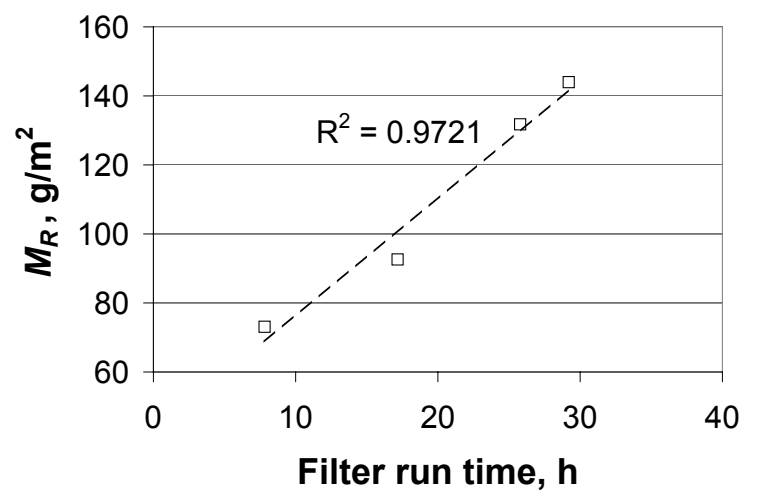

Figure 3

Correlation between mass retained and filter run time for alum coagulation and backwash at $61 \mathrm{~m} / \mathrm{h}$

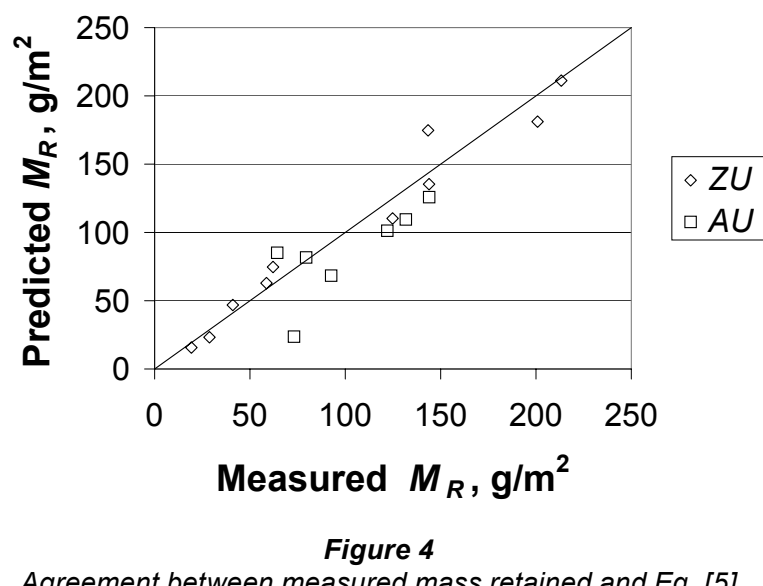

filter at the end of the filter run. Three different measures of the degree of filter clogging: terminal head loss, filter run time and mass deposited (mass retained + mass detached). All three variables were highly correlated with each other and with $M_{R}$ but $M_{R}$ was most strongly correlated with filter run time $\left(\mathrm{R}^{2}=0.97\right.$ as opposed to $\mathrm{R}^{2}=0.96$ for mass deposited and $\mathrm{R}^{2}=0.93$ for terminal head loss). This is consistent with independent experimental results presented in Chapter 8 of Brouckaert (2004) which indicate that floc deposits became more difficult to detach the longer they remained in the filter (an ageing effect). Therefore it appears that run time is not only correlated with the amount of floc deposited in the filter but also with the extent of time-dependent changes in the properties of filter deposits which make them more difficult to remove. The correlation between $M_{R}$ and run time is shown in Fig. 3 .

To the best of the authors' knowledge, the effect of floc ageing on backwash efficiency has not previously been considered in the literature on backwash fundamentals. However, it is has been reported that allowing filters to run for more than $36 \mathrm{~h}$ during periods with low rates of head loss development can result in irreversible deterioration of the filter media, even with auxiliary backwash (Monk and Willis, 1987).

The best choice for $p_{f 1}$ for the $A U$ experiments therefore appeared to be filter run time $\left(t_{f}\right)$. Stepwise regression was used to look for additional parameters $p_{f 2}, \ldots$ which might reflect other variations in floc properties (Table 3 ) but no further significant correlations were found. The final linear regression result for the $A U$ data set is:

$$
\begin{aligned}
& M_{R, A U}=-9.583 v_{b}+3.545 t_{f}+627.46 \\
& R^{2}=0.965
\end{aligned}
$$

Stepwise linear regression was then applied to the data for the $Z U$ experiments. Once again, filter run time was the best choice for $p_{f l}$ and no statistically significant correlations were found with any other variable. The model for the $\mathrm{Z} 464 \mathrm{~N}$ experiments is:

$$
\begin{aligned}
& M_{R, Z U}=-5.953 v_{b}+4.777 t_{f}+350.56 \\
& R^{2}=0.958
\end{aligned}
$$

When the regression coefficients in Eqs. [4] and [5] were compared, it was found that they were not statistically different at the $95 \%$ confidence level. Figure 4 shows the agreement between the predictions for Eq. [5] (calibrated using the $Z U$ data) and the measured values for both the $A U$ and $Z U$ experiments.

Generally, one expects flocs formed using polymeric coagulants or filter aids to be stronger, easier to attach during filtration and more difficult to detach during backwashing than flocs formed using alum. In this case, the filter turbidity removals for the two sets of experiments were very similar ( approximately $90 \%$ ) but the residual mass retained was higher for the $Z U$ experiments than for the $A U$ experiments with the same backwash rates even when the total masses deposited were lower. Figure 4 suggests that the lower backwash efficiencies for the $Z U$ experiments were actually due to longer filter run times (40 to $50 \mathrm{~h}$ as opposed to a maximum of $32 \mathrm{~h}$ in the $A U$ experiments) rather than inherent differences in the floc properties.

The apparently similar behaviour of the two flocs may also have been due in part to the fact that $\mathrm{Z} 464 \mathrm{~N}$ includes an inorganic aluminium component (aluminium chlorohydrate) which produces the same aluminium precipitate as alum (Gebbie, 2005). However, since there was no direct comparison between backwash efficiency for alum and Z464N floc with the same run time, the results presented are inconclusive with respect to the relative strengths of the two flocs. Nonetheless, it is clear that floc ageing effects have a significant impact on backwash efficiency and that more research in this area is needed.

\section{Accumulation of residual deposits over multiple runs}

Figure 5 shows the cumulative mass retained in the $0.7 \mathrm{~mm}$ sand filter as a function of the number of consecutive filter runs with fluidised water backwash. The error bars were calculated based on the uncertainties in the measurement of the volume of water used in the combined air and water backwash step and the uncertainty in the suspended solids measurements.

Figure 5 shows that the total mass of residual deposits 


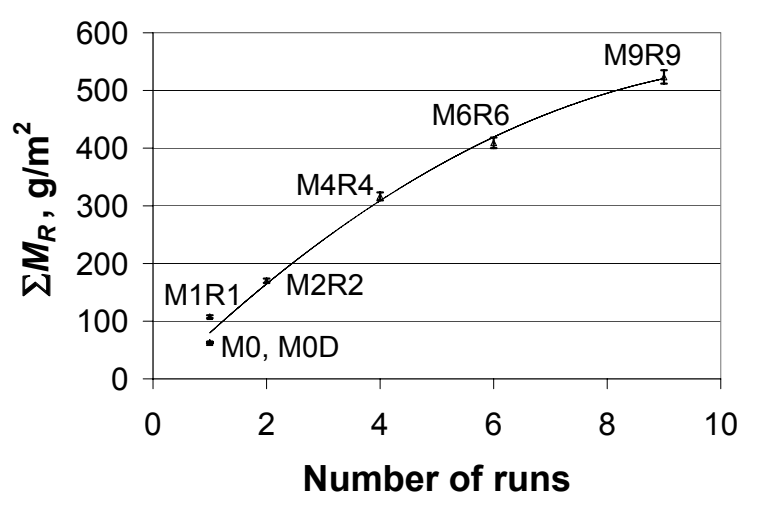

Figure 5

Cumulative mass retained after fluidised backwash

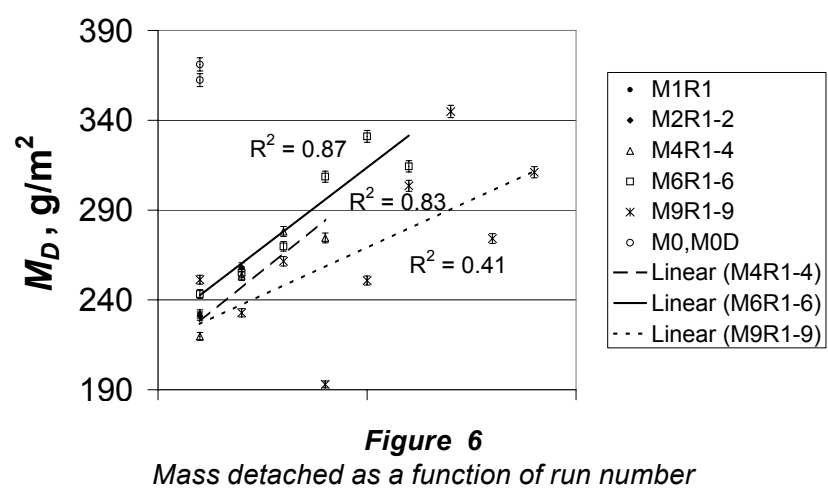

retained in the filter after fluidised backwash increased steadily with increasing number of runs. While the rate of accumulation was not constant, it appears that the incremental increases in mass retained per run were fairly similar. This suggests that:

- If the floc deposited during a given filter run was not detached during the backwash at the end of that particular run, it became increasingly less likely that it would be detached in subsequent backwashes. This allowed the steady accumulation of the total mass of deposits retained

- The proportion of freshly deposited floc detached during backwash is likely to be similar under similar experimental conditions.

Figure 5 also indicates that the rate of accumulation of retained deposits decreased as the number of runs increased. There are a number of possible explanations for this trend. Kawamura (1975) suggested that the accumulation of residual deposits and formation of mud balls is accelerated by the presence of finer grains in the filter bed. As the finer grains become incorporated into mud balls, the effective media size at the top of the bed may increase resulting in a reduced rate of mud retention after backwash. The formation of mud balls also results in increased local velocities during backwashing which could result in more efficient detachment of freshly deposited floc. Finally, as mud accumulates in the bed, the mud balls formed tend to increase in size. The hydrodynamic forces on a solid mass within a fluidised bed increases with the weight of the mass. Consequently, the rate of abrasion of mud balls increases, tending to limit their rate of growth (Baylis, 1954) as well as the overall rate of accumulation of residual mass in the filter.

If the mass increment retained after backwash generally decreases with increasing run number, then this trend should be reflected in the data for individual series. In the mass accumulation experiments (with the exception of M0, M0D and M1R1) the only direct mass measurement for individual runs was the mass detached by water backwashing, $M_{D}$. Figure 6 shows mass detached for individual runs as a function of run number. Trend lines for series M4R1-M4R4, M6R1-M6R6 and M9R1-M9R9 are also shown. Figure 6 shows an overall increase in mass detached with increasing run number for all series, suggesting that the incremental mass retained per run did in fact decrease as each series of experiments progressed.

\section{Effect of filtration conditions on backwash efficiency in the mass accumulation experiments}

Some of the variability in the results of these experiments may also have been due to variations in filtration conditions. The best choice of variable from Table 3 for $p_{f 1}$ in Eq. [3] was found to be the rate of head loss development $\left(d h_{f} / d V_{f}\right)$. The correlation between the average rate of head loss development for each series of experiments and the average mass retained per run is shown in Fig. 7. Figure 7 shows that the rate of head loss development and mass retained per run for the single cycle experiments M0 and M0D were more similar to the 9 run series (M9R1-M9R9) which was conducted at around the same time than to the other single cycle experiment M1R1 which was conducted 5 weeks later. Rate of head loss development did not appear to be correlated with run number. Overall, higher rates of head loss development corresponded to higher rates of residual mass accumulation. This was to some extent expected since the greater the tendency of floc particles to adhere to the filter grains, the more rapidly they would accumulate in the top layers of the media during filtration and hence the greater the rate of head loss development. The following model was therefore proposed:

$$
\begin{aligned}
& \left(\sum M_{R}\right)_{i}=\left(\sum M_{R}\right)_{i-1}+a_{0}+a_{f 1}\left(\frac{d h_{f}}{d v_{f}}\right)_{i}-\beta\left(\sum M_{R}\right. \\
& a_{0}, a_{f 1}, \beta=\text { fitting parameters }
\end{aligned}
$$

A spreadsheet model was set up to calculate the increase in residual mass retained from run to run using Eq. [6]. Initial estimates of the fitting parameters were used to calculate $\Sigma(M R)_{i}$ at the end of each series (M0, M0D, M1R1, M2R2, M4R4, M6R6, M9R9). The sum of squared errors (SSE) was computed and then the spreadsheet model was used to solve for the values of the fitting parameters which minimised SSE. Finally, the significance of each of the model terms was evaluated by testing the following hypotheses: (i) $a_{f 1}=\beta=0$; (ii) $a_{f 1}=0$; (iii) $\beta=0$ using the procedure outlined in Section 10.6 of Walpole and Myers (1985). The results are presented in Table 4.

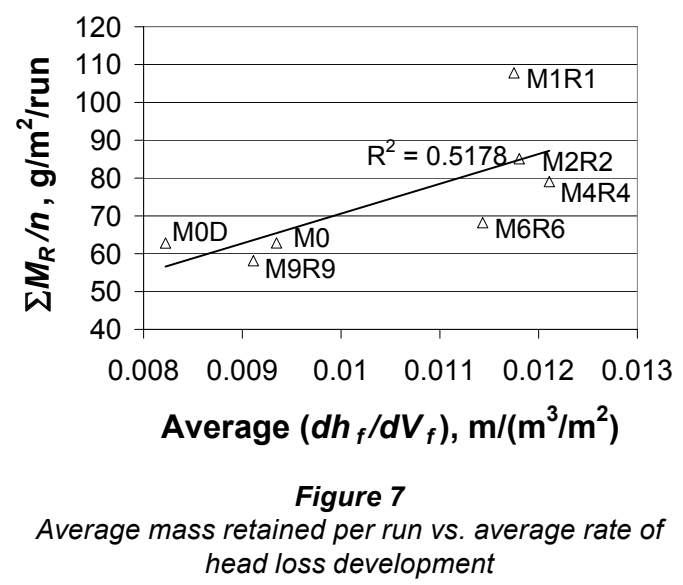

Available on website http://www.wrc.org.za ISSN 0378-4738 = Water SA Vol. 32 No. 5 (Special edn. WISA 2006) ISSN 1816-7950 = Water SA (on-line) 


\begin{tabular}{|l|c|c|c|}
\hline \multicolumn{4}{|c|}{ TABLE 4 } \\
Model significance \\
\hline Null hypothesis $\left(\mathbf{H}_{\mathbf{0}}\right)$ & SSE $\left(\mathbf{H}_{\mathbf{0}}\right)$ & $\mathbf{F}$ statistic & Critical $\mathbf{F}(\alpha=\mathbf{0 . 0 5})$ \\
\hline$a_{f l}=\beta=0$ & 10762 & 20.17 & 6.94 \\
\hline If $\beta \neq 0$ then $a_{f l}=0$ & 1951 & 4.04 & 7.71 \\
\hline If $a_{f l} \neq 0$ then $\beta=0$ & 2786 & 7.48 & 7.71 \\
\hline
\end{tabular}

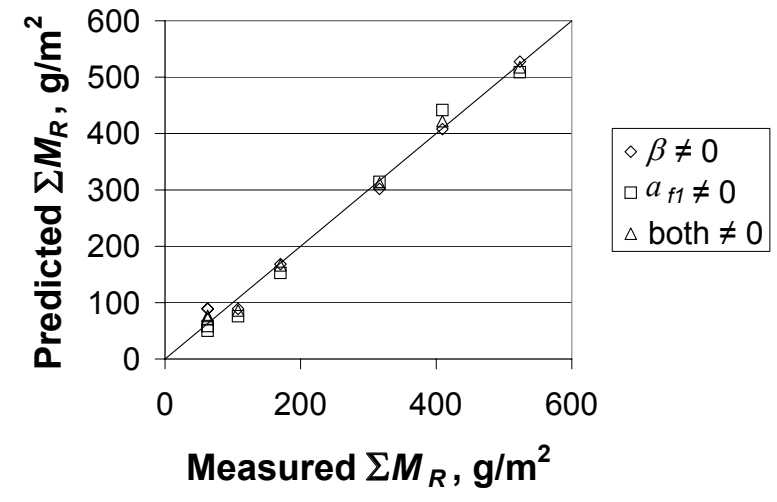

Figure 8

Model performance

\section{Model significance}

SSE $\left(\mathrm{H}_{0}\right)$ is the model sum of squared errors if the null hypothesis is accepted. The agreement between model predictions and measured $(\Sigma M R)_{\mathrm{i}}$ is shown in Fig. 8. Table 4 shows that the inclusion of either $\left(d h_{f} / d V_{f}\right)_{i}$ or $(\Sigma M R)_{i-1}$ in Eq. [6] is significant at the $95 \%$ confidence level. Including $(\Sigma M R)_{i-1}$ instead of $(d h$ $\left./ d V_{f}\right)$ resulted in a lower SSE but meant the model was unable to explain the variability in results for single run experiments (M0, M0D, M1R1)

The apparent anomaly by which $\left(d h_{f} / d V_{f}\right)_{i}$ and $(\Sigma M R)_{i-1}$, which are not significantly correlated with each other, appear to be separately but not jointly significant, is probably the result of the small number of data points used. Therefore, the rate of head loss development may still be a useful predictor of floc deposit strength in some cases. Note that if filters runs are terminated based on head loss as they were for the AU and ZU experiments, mass retained could appear to be negatively correlated with or unrelated to rate of head loss development since lower rates of head loss development would result in longer run times. However, for runs of similar length as was the case for the mass accumulation experiments, the correlation between rate of head loss development and floc deposit strength becomes more apparent.

\section{Developing correlations for backwash efficiency in full-scale filters and filters with auxiliary wash}

It is important to remember that the correlations developed in this study are empirical and site specific. The numerical values of the coefficients in Eqs. [2] and [3] and in many cases, also the variables which should be included in the predictions, will vary from site to site and for different ranges of operating conditions. Therefore, an appropriate version of Eq. [3] has to be developed for each site to which it will be applied.

This will require measurements of the mass retained in filters after backwashing. Estimates of the mass retained in a full scale filter can be obtained from floc retention analysis of core samples extracted from the filter bed. Detailed procedures for filter media sampling and analysis of retained solids are provided in Chapter 10 of Logsdon et al. (2002) and Appendix 8 of Kawamura (1990). The application of floc retention analysis to the prediction of the rate of accumulation of residual mud in filters operating in the field is discussed further in Brouckaert et al. (2006).

Note that analysis of the backwash effluent turbidity profile (Kawamura, 1990, Logsdon et al., 2002) is useful for assessing the efficiency of backwash water usage but cannot be used to estimate the incremental increase in mass retained in the filter after backwash unless both the total mass deposited during filtration and the total mass detached during backwashing can be determined very accurately. In practice, this is seldom the case and the mass retained in the filter media should be measured directly whenever possible.

Many full-scale filters use auxiliary backwash (air scour or surface wash) in addition to or instead of fluidised backwash. The methodology described in this paper can be extended to cases involving auxiliary backwash by identifying parameters which are correlated with the intensity of backwash. These might include air scour rate and/or deviation from the collapse pulsing condition as defined by Amirtharajah (1984) in the case of air scour, surface wash rate and head applied to the bed by water jets in the case of surface wash and/or energy input to the backwash process due to auxiliary wash. Formulae for the calculation of energy input due to auxiliary wash can be found in Kawamura (1990).

Measuring the accumulation of mass retained in filters after backwashing with auxiliary wash will be more challenging than in the case of fluidised backwash because of the smaller concentrations of deposits and lower rates of accumulation involved. It may be necessary to monitor the state of the filter media over many more filter cycles to discern a measurable increase in accumulated solids and/or the rate of accumulation of solids may level off much sooner than in the case of fluidised backwash alone. For small scale experimental plants, the method used for dislodging retained floc in this study (pulsing the air flow) may not be sufficiently vigorous or reproducible for recovering deposits retained after auxiliary wash and alternate methods would have to be explored. Methods for obtaining more reproducible measurements of retained solids in media samples are discussed in Van Staden and Haarhoff (2004).

\section{Conclusions and recommendations}

\section{Factors affecting the efficiency of backwash}

In this study, the age of floc deposits was found to be a major factor determining the efficiency of backwash. This has important implications for both the management of filters and design of experiments to study backwash processes. Treatment plant operators should be aware that long filter run times can negatively impact backwash effectiveness especially with less efficient backwash regimes and should minimise the delay time between the end of filtration and backwash. In experimental 
studies of backwash, the importance of using realistic filter run times should not be overlooked. Some researchers (Fitzpatrick, 1993) have tended to use concentrated influent suspensions to clog filters in relatively short periods of times (e.g. 6 h). However, the backwash performance of a filter clogged within $6 \mathrm{~h}$ is likely to be different from the behaviour of a filter that reaches the same terminal head loss or mass of deposits over $24 \mathrm{~h}$. The rate of head loss development was found to be a useful indicator of the strength of cohesive deposits for filter runs of similar length.

\section{Accumulation of residual deposits over multiple filter cycles}

Backwash efficiency was found to decrease the longer the floc deposits remained in the filter, and it was concluded that the deposits which survived one backwash would be unlikely to be removed in later backwashes. Experimental results indicated a fairly steady increase in mass retained in the filter with increasing number of consecutive runs for up to 9 runs. The rate of accumulation did however tend to decrease as the amount of residual mud in the filter increased. This may have been due to the change in size distribution of the media in the top section of the filter as the finest grains became encapsulated in mud balls. However, the abrading forces experienced by mud balls during backwashing would tend to increase with mud ball size and this may also have been a factor. More research is required to study this phenomenon for greater numbers of filter cycles and different filter media designs.

\section{Implications for backwash modelling}

The results of this study demonstrate that it is possible to make quantitative predictions about backwash efficiency based on data that should be readily available at most filtration plants. The numerical correlations developed are site- and filter-specific but the methodology can be adapted to any filter operation.

While this investigation was limited to fluidised bed backwash without air scour or surface wash, the general approach is equally applicable to the more technologically relevant case of backwash with auxiliary wash. In the case of auxiliary backwash, one would simply need to identify additional parameters which are correlated with the intensity of backwash.

The simple linear correlations developed here provide an important first step towards developing and calibrating a more comprehensive model of backwash efficiency that could easily be integrated with existing models of filtration. More research is required to extend the model's applicability to a wider range of filtration and backwash conditions. In particular, this research pointed to the likely importance of filter deposit ageing, filter grain size distribution and mud-balling effects in determining backwash efficiency.

\section{Acknowledgements}

This research was partially funded by the South African Water Research Commission. Experimental facilities were provided by Umgeni Water. The assistance of the staff of the Umgeni Water Process Evaluation Facility, in particular, Mr Martin Pryor and Mr Rachi Rajagopaul, is gratefully acknowledged.

\section{References}

AMIRTHARAJAH A (1978) Optimum backwashing of sand filters. J. Environ. Eng. Div. Am. Soc. Civ. Eng. 104 (10) 917-932.

AMIRTHARAJAH A (1984) Fundamentals and theory of air scour J. Environ. Eng. Div. Am. Soc. Civ. Eng. 110 (3) 573-590.

AMIRTHARAJAH A and GIOURGAS AJ (1981) Theory for particle detachment from sand grains during backwashing of filters. Int. Symp. on Appl. Fluid Mechanics and Heat Transfer for Energy and Environ. Eng., University of Patras, Greece.

BAYLIS JR (1954) Washing and maintenance of filters. J. Am. Water Works Assoc. 46 (2) 176-186.

BROUCKAERT BM (2004) Hydrodynamic Detachment of Deposited Particles in Fluidised Bed Backwashing. Ph.D. Thesis. Georgia Institute of Technology, Atlanta. Also available at http://etd.gatech. edu/theses/available/etd-07082004-115123

BROUCKAERT BM, AMIRTHARAJAH A, RAJAGOPAUL R and THOMPSON P (2006) Filter backwash options for rural treatment plants. Proc. 10 $0^{\text {th }}$ Bienn. Conf. of the Water Inst. of Southern Africa. Durban, May.

CLEASBY JL (1990) Filtration. Chapter 8 in Water Quality and Treatment $\left(4^{\text {th }}\right.$ edn.). McGraw-Hill, Inc., New York. 455 pp.

FITZPATRICK CSB (1993) Observations of particle detachment during filter backwashing. Water Sci. Technol. 37 (10) 213-221.

GEBBIE P (2005) A dummy's guide to coagulants. Proc. $68^{\text {th }}$ Annual Water Ind. Engineers and Operators' Conf., Bendigo, Australia. $75 \mathrm{pp}$.

HALL D and FITZPATRICK CSB (2000) Suspension concentration profiles during rapid filter backwashing. Chem. Eng. J. 80 197-201.

KAWAMURA S (1975) Design and operation of high-rate filters - Part 1. J. Am. Water Works Assoc. 67 (10) 535-544.

KAWAMURA S (1990) Integrated Design of Water Treatment Facilities. John Wiley and Sons, Inc., New York. 248 pp.

LOGSDON GS, HESS AF, CHIPPS MJ and RACHWAL AJ (2002) Filter Maintenance and Operations Guidance Manual. AWWA Research Foundation and American Water Works Association, Denver. 6-4 pp.

MAHMOOD T, AMIRTHARAJAH A, STURM TW and DENNET KE (1998) A micromechanics approach for attachment and detachment of asymmetric colloidal particles. Proc. $72^{\text {nd }}$ Am. Chem. Soc. Colloids Surf. Sci. Symp. Penn State University.

MONK RDG and WILLIS JF (1987) Designing water treatment facilities. J. Am. Water Works Assoc. 79 (2) 45-57.

STANDARD METHODS (1990) Standard Methods for the Examination of Water and Wastewater. American Public Health Association/ American Water Works Association/Water Environmental Federation, Washington DC

VAN STADEN SJ and HAARHOFF J (2004) A standard test for filter media cleanliness. Water $S A 30$ (1) 81-88.

WALPOLE RE and MYERS RH (1985) Probability and Statistics for Engineers and Scientists. Macmillan Publishing Company, New York. 373 pp. 